\title{
Avaliação físico-química e tecnológica do suco de sete cultivares de macieira
}

\section{Physical chemical and technological evaluation of the juice of seven apple tree cultivars}

\author{
Alessandro Nogueira ${ }^{1}$; Inara Biscaia ${ }^{2}$; Flávia Villas Boas Wiecheteck ${ }^{2}$; \\ Frederico Denardi ${ }^{3}$; Gilvan Wosiacki ${ }^{*}$
}

Resumo

\begin{abstract}
A pomicultura, concentrada na Região Sul, é responsável por $98 \%$ da produção brasileira. Cerca de $80 \%$ desta produção (880 mil toneladas), são destinadas ao consumo in natura e em torno de 220 mil toneladas representam o descarte comercial, utilizado pelo setor industrial. Devido ao aumento gradativo da produção, a composição destas frutas passa a ser de interesse, tanto comercial quanto industrial. Tendo isto em foco, foram feitas análises físico-químicas de cinco cultivares comerciais e duas seleções avançadas. Os valores médios das cultivares apresentaram diferenças significativas em todas as análises realizadas, salvo sobre o teor de ácidos, onde os valores não se diferenciaram ao nível de $5 \%$. Os resultados demonstraram que os açúcares redutores totais variaram de 10,48 a 12,94 g/100mL para as cultivares Daiane e Sansa, respectivamente. A razão entre açúcares totais e acidez apresentou valores superiores ao critério 20, devido ao baixo teor de acidez total, todos inferiores a $0,38 \mathrm{~g} / 100 \mathrm{~mL}$, o que acarreta pouco interesse para o setor de transformação. As cultivares apresentaram teores de fenóis inferiores a $459 \mathrm{mg} / \mathrm{L}$, salvo a cv. Sansa que apresentou o maior teor de fenóis $(624 \mathrm{mg} / \mathrm{L})$, que pode ser utilizada para cortes ou blends.
\end{abstract}

Palavra-chave: Malus domestica, suco, indicadores industriais

\begin{abstract}
The pomiculture in the Southern States of Brazil is responsible for $98 \%$ of the Brazilian apple production. Up to $80 \%$ of this production, (880 metric tons), are sold as fresh fruit and around 220 metric tons are apple with low commercial appeal, being utilized by industrial sector. Due to stepwise increase of apple production, the fruit composition becomes important both for commercial and processing sectors. To prepare this paper, five cultivars and two advanced selection were analyzed. The average results showed that almost all samples were statistically different at $5 \%$ level of significance but only acidity was not. Total sugars range was from 10.48 to $12.94 \mathrm{~g} / 100 \mathrm{~mL}$ for Daiane and Sansa cultivars, respectively. The indicator sugar/acidity ratio were quite high compared to the criteria used (index $=20$ ), meaning that all samples have a low acid content, being of low fitness for processing. The cv. Sansa has the highest content of phenol compounds $(624 \mathrm{mg} / \mathrm{L})$, while all the other cultivars have less than $459 \mathrm{mg} / \mathrm{L}$. The analyzed samples showed low total acidity and phenol compounds and only the cv Sansa seems to be feasible for use as blending.
\end{abstract}

Key words: Malus domestica, juice, industrial indicators

1 Engenheiro Agrônomo, Doutor em Processos Biotecnológicos Agroindustriais, bolsista Recém-doutor do CNPq.

2 Acadêmica de Engenharia de Alimentos, bolsista de Iniciação Cientifica do CNPq.

3 Engenheiro Agrônomo, MSc.em Fruticultura, Pesquisador da Estação Experimental de Caçador da EPAGRI/SC.

4 Engenheiro Químico, Mestre em Bioquímica, Doutor em Ciência dos Alimentos, Pós-doutorado em Tecnologia de Frutas e Hortaliças. Departamento de Engenharia de Alimentos, Setor de Ciências Agrárias e de Tecnologia, Universidade Estadual de Ponta Grossa, Ponta Grossa PR E-mail: wosiacki@uol.com.br

* Autor para correspondência.

Recebido para publicação 01/03/05 Aprovado em 25/08/05 


\section{Introdução}

A cultura da maçã destacou-se entre as frutas de clima temperado por ter apresentado a maior expansão nos últimos 30 anos. De um total de 931 ha plantados em 1972 passou para 18.041 ha em 1980. Atualmente, existem mais de 30.000 ha cultivados com macieiras no país. A produção de maçãs, localizada principalmente na Região Sul do Brasil, atingiu na safra de 2003/2004 a cifra 989.961 mil ton de frutas (ASSOCIAÇÃO BRASILEIRA DE PRODUTORES DE MAÇA, 2005). Esta produção é composta por cultivares comerciais produtoras e polinizadoras, ambas melhoradas e selecionadas para atender ao mercado in natura (WOSIACKI; NOGUEIRA, 2005).

As principais cultivares brasileiras em termos de área plantada e produção são a Gala (46\%), a Fuji (45\%) e a Golden Delicious (4\%), que juntas correspondem a $95 \%$ da produção total. As características destas três cultivares já foram bem discutidas na literatura especializada (NAMIUCHI; WOSIACKI; ROCHA, 1992; DENARDI; CAMILO, 1997b; CAMILO; DENARDI, 2002; WOSIACKI et al., 2002; CZELUSNIAK et al., 2003), porém existem outras cultivares comerciais que apresentam baixo destaque na produção, ou seja, pouco conhecidas e que podem apresentar características interessantes para o setor agroindustrial.

$\mathrm{Na}$ comercialização das maçãs, a etapa de classificação pode gerar um descarte de até 30\% (KENNEDY et al., 1999), que é destinado ao setor industrial, onde os principais produtos são o suco, a sidra, destilados e o vinagre. Porém este descarte, basicamente de 'Gala' e 'Fuji', não apresenta características químicas adequadas, como elevados teores de acidez e fenóis, principalmente os taninos, desejados na qualidade dos produtos. No caso da sidra, já na década de 50, trabalhos como o de Smock e Neubert (1950) indicavam uma perda importante do "corpo" da sidra quando feita apenas com maçãs de consumo in natura.

O setor agroindustrial da maçã, que já domina a tecnologia e o savoir faire de vários produtos, busca atualmente a melhoria da qualidade e dá início a uma demanda por frutas com características específicas para o processamento, que possam beneficiar a qualidade final do produto.

Assim, face ao gradual aumento da produção de maçãs e, consequentemente, da parcela conhecida como industrial, o conhecimento acerca da sua qualidade passa a ser de interesse, tanto comercial quanto do setor agroindustrial. Desta forma, este artigo teve como objetivo levantar na literatura as características agronômicas, fenológicas e a características das frutas; e avaliar a composição físico-química de 5 cultivares brasileiras de maçãs comerciais e duas ainda em processo de melhoramento genético.

\section{Material e Métodos}

\section{Material}

Foram analisadas quatro cultivares de maçãs comerciais: Baronesa, Daiane, Fred Hough e Imperatriz desenvolvidas pela Estação Experimental de Caçador/Epagri, SC; e duas seleções avançadas ainda em processo de avaliação agronômica codificadas como M-11/00 e M-13/00. A cv. Sansa foi desenvolvida pela Estação de Fruticultura de Morioka, no Japão e implantada no Brasil pela EPAGRI. As amostras foram fornecidas pela EPAGRI, sendo que as frutas foram colhidas na safra de 2003/2004 em estádio de maturação para consumo, na fase de meia estação de colheita, por pessoal treinado. Produtos químicos foram de qualidade pro analise e as enzimas comerciais, cedidas pela NOVOZYMES do Brasil.

\section{Métodos}

As frutas de cada cultivar $(5 \mathrm{~kg})$ foram processadas a fim de obter o suco clarificado, sendo selecionadas e lavadas, trituradas e prensadas durante 5 minutos a $3 \mathrm{kgf} / \mathrm{cm}^{2}$. Ao suco bruto, foi adicionado pectinase (Batch 1201371 L, Pectinex 100 L, Novozymes do Brasil) $3 \mathrm{~mL} / \mathrm{hL}$, e após 2 horas a 
$45^{\circ} \mathrm{C}$ o sobrenadante foi trasfegado para garrafas, fechadas hermeticamente e submetidas a um tratamento térmico de estabilização $\left(85^{\circ} \mathrm{C} / 20 \mathrm{~min}\right.$. $)$, conforme recomendado por Wosiacki et al. (1989). No suco estabilizado, foram avaliados os parâmetros físico-químicos em triplicatas. Os açúcares foram determinados por Somogyi-Nelson, segundo métodos oficiais (TANNER; BRUNNER, 1985), sendo a glucose determinada especificamente pelo método enzimático colorimétrico da glucose oxidase (Gold Analisa Diagnóstica, Glicose - PP). A sacarose foi determinada por diferença entre os açúcares redutores totais e solúveis e a frutose através da diferença entre os açúcares redutores solúveis e a glucose, sendo todos os açúcares expressos como monossacarídeo, glucose, em g/100 mL. A acidez total titulável foi expressa como ácido málico em g/ $100 \mathrm{ml}$ (IAL, 1976), já que este ácido é predominante em maçãs e os demais ácidos orgânicos não chegam a atingir o valor correspondente a 5\% do total, razão pela qual são usualmente negligenciados (CZELUSNIAK et al., 2003). A determinação da intensidade da cor foi calculada como a somatória das absorvâncias a 440 e $520 \quad \mathrm{~nm} \quad$ em espectrofotômetro (Femto, modelo 435), sendo a primeira correspondendo a oxidação dos polifenóis e a segunda aos pigmentos antocianínicos da fruta (TANNER; BRUNNER, 1985). Os compostos fenólicos totais foram determinados com o reativo de Folin-Ciocalteau e expressos em ppm de catequina (SINGLETON; ORTHOFER; LAMUELARAVENTOS, 1999; ASSOCIATION OF OFICIAL ANALYTICAL CHEMISTS, 1965). Os compostos fenólicos e a acidez total titulável foram também utilizados como marcadores de qualidade na classificação das amostras, com relação ao seu potencial de industrialização. O nitrogênio foi determinado através do método de Kjeldhal e expresso em mg de nitrogênio/L. Os resultados foram avaliados mediante estatística simples, pela análise de variância (ANOVA) e a diferenciação das médias pelo teste de Tukey em nível de 5\%, a fim de verificar e identificar possíveis diferenças significativas entre os tratamentos realizados (DUTCOSKI, 1996).

\section{Resultados e Discussão}

$\mathrm{Na}$ Tabela 1 estão apresentadas as características agronômicas, dados fenológicos e qualidade das frutas utilizadas neste estudo. Pode ser observado que a cultivar Sansa apresenta a maior exigência de frio $(>800 \mathrm{~h})$ o que pode influenciar o local de implantação do pomar. Em comparação com as cultivares desenvolvidas pela EPAGRI a cv. Sansa é a mais precoce, com maturação final em 05/02 e tem alta produtividade, porém apresenta baixa conservação (1 mês de conservação em atmosfera controlada), ou seja a produção deve ser rapidamente comercializada. Nas características de cada cultivar, podem ser observadas, além de diferenças da Sansa com as demais, diferenças entre as outras quatro cultivares (Daiane, Imperatriz, Baronesa e Fred Hough). Desta forma, as cultivares comerciais e as seleções avançadas foram analisadas a fim de observar possíveis diferenças na composição química.

Os resultados médios das análises físico-químicas das 7 amostras de maçãs estão apresentados na Tabela 2. As médias dos açúcares redutores totais (ART), entre as cultivares analisadas, apresentaram diferença significativa ao nível de 5\%. A cv. Sansa foi a que teve o maior valor $(12,94 \mathrm{~g} / 100 \mathrm{~mL})$, enquanto a 'Daiane', a 'Baronesa' e a 'Fred Hough' apresentaram os menores valores, sem diferença significativa entre si $(10,48 ; 10,56$ e 10,81 g/100 mL, respectivamente), conferindo com os valores médios das maçãs européias, de 10,60 g/100 mL (FEALTHE, 1987). Em termos de açúcares redutores solúveis (ARS), as médias também foram diferentes ao nível de 5\%; entretanto, o maior valor foi o da cv. Daiane $(10,48 \mathrm{~g} / 100 \mathrm{~mL})$, o mesmo valor de ART devido ao baixo teor de sacarose, insuficiente para a sensibilidade da metodologia de quantificação utilizada. As cultivares Baronesa e Sansa tiveram o segundo maior teor $(9,00$ e $8,83 \mathrm{~g} / 100 \mathrm{~mL})$, semelhante aos valores médios do RSK europeu $(9,10 \mathrm{~g} / 100 \mathrm{~mL})$. A seleção M-11/00 apresentou o menor valor de ARS $(5,67 \mathrm{~g} / 100 \mathrm{~mL})$, indicando baixos teores de glucose e frutose. 
Tabela 1. Características das cultivares comerciais de macieira estudadas.

\begin{tabular}{|c|c|c|c|c|c|}
\hline \multirow[t]{2}{*}{ Características } & \multicolumn{5}{|c|}{ Cultivares } \\
\hline & SANSA & DAIANE & IMPERATRIZ & BARONES A & FRED HOUGH \\
\hline \multicolumn{6}{|c|}{ Car acterísticas agronômic as e dados fenológicos } \\
\hline \multicolumn{6}{|c|}{ Características da planta } \\
\hline Porte da copa & Pouco-vigoroso & Semi-vigoroso & Semi-vigoroso & Vigoroso & Semi-anão \\
\hline Hábito vegetativo & Semi- aberto & Fechado & Semi-aberto & Semi-aberto & Fechado \\
\hline $\begin{array}{l}\text { Exigência de Frio } \\
(\mathrm{h})^{1 /}\end{array}$ & $>800$ & $650-750$ & $600-650$ & $500-600$ & $600-650$ \\
\hline \multicolumn{6}{|l|}{ Reação a doenças } \\
\hline Sarna & Resistente & Suscetível & Susceptível & Pouco susceptível & Muito resistente \\
\hline Oíd io & Susceptível & $\begin{array}{l}\text { Resistência } \\
\text { méd ia }\end{array}$ & Pouco susceptível & Pouco susceptível & Muito susceptível \\
\hline Podridão a marga & Suscetível & Suscetível & Pouco susceptível & Susceptível & Susceptível \\
\hline $\begin{array}{l}\text { Mancha foliar da } \\
\text { Gala }\end{array}$ & Resistente & Resistente & Resistente & Resistente & Suscetível \\
\hline $\begin{array}{l}\text { Precocidade } \\
\text { frutífera }\end{array}$ & Alta & Média a alta & Média & Muito alta & Alta \\
\hline \multicolumn{6}{|l|}{ Dados fenológicos } \\
\hline Início brotação & $02 / 10$ & $07 / 10$ & $20 / 09$ & $17 / 09$ & $17 / 09$ \\
\hline \multicolumn{6}{|l|}{ Floração } \\
\hline Início & $05 / 10$ & $10 / 10$ & $23 / 09$ & $20 / 09$ & $20 / 09$ \\
\hline Plena & $15 / 10$ & $20 / 10$ & $05 / 10$ & $30 / 09$ & $30 / 09$ \\
\hline Final & $30 / 10$ & $30 / 10$ & $20 / 10$ & $10 / 10$ & $17 / 10$ \\
\hline \multicolumn{6}{|c|}{ Matur ação dos frutos } \\
\hline Início & $20 / 01$ & $05 / 03$ & $25 / 01$ & $28 / 03$ & $25 / 02$ \\
\hline Final & $05 / 02$ & $20 / 03$ & $10 / 02$ & $15 / 04$ & $10 / 03$ \\
\hline Produtividade & Alta & Média a alta & Média & Muito alta & Alta \\
\hline \multicolumn{6}{|c|}{ Características dos frutos } \\
\hline Cor ep iderme & $\begin{array}{l}\text { Vermelho- } \\
\text { brilhante }\end{array}$ & $\begin{array}{l}\text { Vermelho- } \\
\text { estriada }\end{array}$ & $\begin{array}{c}\text { Vermelho- } \\
\text { estriada }\end{array}$ & Vermelho-opaca & Vermelho-estriada \\
\hline Cor de fundo & $\begin{array}{l}\text { Amarelo- } \\
\text { esverdeado }\end{array}$ & Amarela & Amarela & Verde-a ma relada & Amarela \\
\hline $\begin{array}{ll}\begin{array}{l}\text { Formato } \\
\text { frutos }\end{array} & \\
\end{array}$ & Redondo cônicos & Cônico & Oblongo-cônicos & Arredondados & Redondo cônicos \\
\hline Peso médio (g) & 120,0 & 125,0 & 150,0 & 130,0 & 135,0 \\
\hline Pedúnculo & Médio & Médio & Curto e espesso & Curto e méd io & Curto e méd io \\
\hline Cor da polpa & Branca & Amarelo-creme & Amarelo-creme & Branco-creme & Amarelo-creme \\
\hline $\begin{array}{l}\text { Firmeza polpa } \\
\left(\mathrm{lb} / \mathrm{cm}^{2}\right)\end{array}$ & 16,2 & 15,0 & 18,0 & 17,0 & 15,6 \\
\hline Russeting* & Alta & Baixa & Média & Ausente & Ausente \\
\hline Bitter pit* & Alta & Baixa & Ausente & Média & Alta \\
\hline \multicolumn{6}{|c|}{ Conservação (meses) } \\
\hline $\begin{array}{l}\text { Atmosfera } \\
\text { comum }\end{array}$ & 1 & 4 & 5 & 6 & 3 \\
\hline $\begin{array}{l}\text { Atmosfera } \\
\text { controlada }\end{array}$ & - & 6 & - & 10 & 10 \\
\hline $\begin{array}{c}\text { Frutos de cada } \\
\text { uma das } \\
\text { cultivares } \\
\text { estudadas }\end{array}$ & 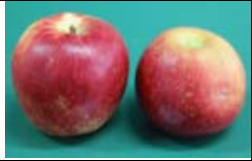 & 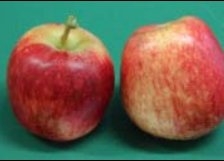 & & & \\
\hline
\end{tabular}

Nota: * incidência.

Fonte: Denardi e Camilo,1994; 1997a; 1997b; 1998; 2000; Camilo e Denardi, 2002.

${ }^{1 /}$ Valores estimados $\leq 7,2^{\circ} \mathrm{C}$, com base no índice de brotação em relação às cultivares padrão Gala e Fuji no Meio Oeste Catarinense. 
Tabela 2. Comparação entre as características físico-químicas de maçãs de quatro novas cultivares e duas seleções avançadas com as maçãs da cv. Sansa procedentes da Estação Experimental de Caçador/Epagri durante a safra 2003/04.

\begin{tabular}{l|c|c|c|c|c|c|c|c|c|c|c}
\hline Cultivares & $\begin{array}{c}\text { ART, } \\
\mathrm{g} / 100 \mathrm{~mL}\end{array}$ & $\begin{array}{c}\text { ARS, } \\
\mathrm{g} / 100 \mathrm{~mL}\end{array}$ & $\begin{array}{c}\text { FRU, } \\
\mathrm{g} / 100 \mathrm{~mL}\end{array}$ & $\begin{array}{c}\text { GLU, } \\
\mathrm{g} / 100 \mathrm{~mL}\end{array}$ & $\begin{array}{c}\mathrm{SAC}, \\
\mathrm{g} / 100 \mathrm{~mL}\end{array}$ & $\begin{array}{c}\text { FRU/ } \\
\text { GLU }\end{array}$ & $\begin{array}{c}\text { Acidez, } \\
\mathrm{g} / 100 \mathrm{~mL}\end{array}$ & $\begin{array}{c}\text { ART/ } \\
\text { Acidez }\end{array}$ & $\begin{array}{c}\text { Cor* } \\
\text { Fenóis, } \\
\mathrm{mg} / \mathrm{L}\end{array}$ & $\begin{array}{c}\text { Nitrogênio, } \\
\mathrm{mg} / \mathrm{L}\end{array}$ \\
\hline Sansa & $\mathbf{1 2 , 9 4} \mathbf{a}$ & $8,83 \mathrm{~b}$ & $6,77 \mathrm{~b}$ & $\mathbf{2 , 0 6} \mathbf{a}$ & $4,12 \mathrm{~cd}$ & $3,29 \mathrm{c}$ & $0,28 \mathrm{a}$ & $46,01 \mathrm{ab}$ & $0,39 \mathrm{ab}$ & $\mathbf{6 2 3 , 7 6} \mathbf{a}$ & $122,91 \mathrm{bc}$ \\
Daiane & $10,48 \mathrm{c}$ & $\mathbf{1 0 , 4 8} \mathbf{a}$ & $\mathbf{8 , 9 6} \mathbf{a}$ & $1,53 \mathrm{~cd}$ & - & $\mathbf{5 , 8 6} \mathbf{a}$ & $0,34 \mathrm{a}$ & $31,54 \mathrm{~b}$ & $0,22 \mathrm{~b}$ & $388,99 \mathrm{c}$ & $123,21 \mathrm{bc}$ \\
Imperatriz & $12,00 \mathrm{ab}$ & $7,50 \mathrm{c}$ & $5,90 \mathrm{c}$ & $1,60 \mathrm{c}$ & $4,50 \mathrm{c}$ & $3,68 \mathrm{c}$ & $\mathbf{0 , 3 8} \mathbf{a}$ & $31,65 \mathrm{~b}$ & $0,40 \mathrm{ab}$ & $459,89 \mathrm{~b}$ & $87,62 \mathrm{~d}$ \\
Baronesa & $10,56 \mathrm{c}$ & $9,00 \mathrm{~b}$ & $7,26 \mathrm{~b}$ & $1,74 \mathrm{~d}$ & $1,56 \mathrm{e}$ & $4,16 \mathrm{~b}$ & $0,30 \mathrm{a}$ & $36,39 \mathrm{ab}$ & $\mathbf{0 , 5 2} \mathbf{a}$ & $347,35 \mathrm{~d}$ & $104,06 \mathrm{~cd}$ \\
Fred Hough & $10,81 \mathrm{c}$ & $6,76 \mathrm{~cd}$ & $5,35 \mathrm{c}$ & $1,40 \mathrm{~d}$ & $4,05 \mathrm{~b}$ & $3,81 \mathrm{bc}$ & $0,20 \mathrm{a}$ & $\mathbf{5 7 , 9 6} \mathbf{a}$ & $0,37 \mathrm{ab}$ & $201,83 \mathrm{f}$ & $142,63 \mathrm{~b}$ \\
M-11/00 & $11,33 \mathrm{bc}$ & $5,67 \mathrm{e}$ & $3,90 \mathrm{e}$ & $1,77 \mathrm{~b}$ & $5,67 \mathrm{~b}$ & $2,21 \mathrm{~d}$ & $0,31 \mathrm{a}$ & $37,12 \mathrm{ab}$ & $0,35 \mathrm{ab}$ & $279,66 \mathrm{e}$ & $\mathbf{1 7 3 , 9 4} \mathbf{a}$ \\
M-13/00 & $12,40 \mathrm{ab}$ & $6,20 \mathrm{de}$ & $4,68 \mathrm{~d}$ & $1,49 \mathrm{~d}$ & $\mathbf{6 , 2 0} \mathbf{a}$ & $3,13 \mathrm{c}$ & $0,29 \mathrm{a}$ & $43,64 \mathrm{ab}$ & $0,24 \mathrm{~b}$ & $192,66 \mathrm{f}$ & $160,37 \mathrm{ab}$ \\
RSK** & 10,60 & 9,10 & 6,50 & 2,60 & 1,50 & 2,50 & 0,65 & 16,30 & - & 490,00 & - \\
\hline
\end{tabular}

Nota: ART: açúcar redutor total; ARS: açúcar redutor solúvel; RSK: Richtwerte und Schwankungsbreiten bestimmenter Kennzahlen mit überarbeiteten Analysenmethoden 1987. *: adimensional; **valores médios europeus. As letras iguais não diferem significativamente ao nível de $5 \%(p>0,05)$.

Com relação ao açúcar frutose, a cv. Daiane foi o destaque, com 8,96 g/100 mL, seguida pelas cultivares Baronesa e Sansa (7,26 e 6,77 g/100 mL), as quais não apresentaram diferença significativa ao nível de 5\%, ficando acima do valor médio do RSK $(6,50 \mathrm{~g} / 100 \mathrm{~mL})$. Entretanto, o RSK preconiza o valor médio para a glucose de 2,60 g/100 mL, que é superior ao encontrado em todas as cultivares analisadas, sendo que a cv Sansa apresentou o maior valor, de $2,06 \mathrm{~g} / 100 \mathrm{~mL}$. Os demais valores, por mais próximos que aparentam estar, foram diferentes ao nível de $5 \%$. Na determinação da sacarose, a seleção M-13/ 00 teve o maior valor $(6,20 \mathrm{~g} / 100 \mathrm{~mL})$, bem superior aos 1,50 g/100 mL do RSK alemão (Tabela 2). Em termos de relação 'frutose/glucose', a cv. Daiane foi que apresentou o maior valor $(5,86)$, seguida da cv. Baronesa com 4,16. Quanto maior esta relação, maior é o teor de frutose que pode ser utilizada na elaboração de alimentos em dietas de ingestão controlada de açúcares (AGÊNCIA NACIONAL DE VIGILÂNCIA SANITÁRIA, 2002).

O teor de acidez total, expresso em ácido málico, foi à única análise que não apresentou diferença significativa ao nível de $5 \%$. Além disso, pode ser observado na Tabela 2, que a acidez das maçãs das cultivares brasileiras é duas vezes menor que a média da européia destinada ao consumo in natura. $\mathrm{O}$ mercado brasileiro prefere maçãs mais doces que as das cultivares tradicionais desenvolvidas no Hemisfério Norte. Desta forma, em países da Europa, o descarte comercial pode ser utilizado pela indústria, juntamente com frutas industriais, uma vez que não vai alterar de forma importante o teor de acidez de sucos e mostos (DEL CAMPO et al., 2004).

A cor dos sucos apresentou diferenças significativas entre a 'Baronesa' $(0,52)$, a cv. Daiane e seleção M-13/00 (0,22 e 0,24, respectivamente); porém, estes valores não apresentam correlação com o teor de compostos fenólicos. A cv. Sansa apresentou o maior valor de fenóis $(623 \mathrm{mg} / \mathrm{L})$, ficando acima da média do RSK, de $490 \mathrm{mg} / \mathrm{L}$, mas principalmente da média nacional, de $300 \mathrm{mg} / \mathrm{L}$ (NOGUEIRA et al., 2004). Esta cultivar, devido aos seus valores de compostos fenólicos, pode influenciar no corpo do suco ou da sidra. Desta forma, parece ser uma boa opção para o processamento ou realização de cortes com mostos pobres nestes compostos.

O nitrogênio é considerado um elemento que pode ser limitante para a levedura na fermentação alcoólica. Segundo Nogueira e Wosiacki (2005), 
mostos com valores abaixo de $60 \mathrm{mg} / \mathrm{L}$ são considerados pobres para o processo fermentativo; de 60 a 120 os valores são adequados e acima de $120 \mathrm{mg} / \mathrm{L}$ possivelmente permanecerá um residual do nutriente após o término da fermentação; isto se não forem realizadas eliminações de biomassa ou adições de oxigênio no início da fermentação. Como pode ser observado na Tabela 2, as cultivares comerciais, por mais que tenham apresentado diferença significativa entre as médias, possuem um teor de nitrogênio dentro da normalidade para o processo fermentativo. Já para as duas seleções avançadas, o teor do nutriente foi superior às demais, fato este que pode ser observado quando as frutas são provenientes de pomares jovens (BARON; BOHUON; DRILLEAU, 1977).

Os resultados das análises físico-químicas, tratadas estatisticamente, estão apresentados na Tabela 3. Os valores encontrados de açúcares totais tiveram um valor médio de $11,51 \mathrm{~g} / 100 \mathrm{~mL}$, com desvio padrão de $0,96 \mathrm{~g} / 100 \mathrm{~mL}$, sendo que o coeficiente de variação encontrado foi de 8,37\%. Em relação aos açúcares redutores solúveis, as cultivares apresentaram uma média de 7,78 $\pm 1,73 \mathrm{~g} / 100 \mathrm{~mL}$, com um coeficiente de variação de $22,27 \%$, o qual foi mais de duas vezes superior ao coeficiente do ART, ou seja, existe uma variação importante no teor dos açúcares redutores (frutose, glucose e sacarose) em função da cultivar.

Dos açúcares redutores totais presentes na maçã, a glucose é uma fração minoritária, tendo sido encontrado um valor médio de 1,66 $\pm 0,22 \mathrm{~g} / 100 \mathrm{~mL}$, o que representa um coeficiente de variação da ordem de $13,28 \%$. Isto é esperado, visto ser a maçã uma fruta com valores expressivos de frutose. Com relação a este monossacarídeo, os valores encontrados apresentaram um valor médio de 6,12 \pm $1,17 \mathrm{~g} / 100 \mathrm{~mL}$, com um coeficiente de variação da ordem de $27,85 \%$. A razão entre estes dois monossacarídeos é importante no aspecto qualificação nutricional da fruta. Apresentou um valor médio de $3,74 \pm 1,13$, com um coeficiente de variação mais elevado, da ordem de $30,17 \%$. Ou seja, cada molécula de glucose neste conjunto corresponde a 2,87 moléculas de frutose.

Tabela 3. Estatística descritiva dos valores médios dos nove parâmetros físico-químicos analisados em amostras de maçãs das cultivares Sansa, Daiane, Imperatriz, Baronesa e Fred Hough e das seleções M-11/00 e M-13/00.

\begin{tabular}{l|c|c|c|c|c|c|c}
\hline \multirow{2}{*}{ Análises } & & \multicolumn{3}{c}{ Valores } \\
\cline { 2 - 8 } & $\mathrm{N}$ & Mínimo & Média & Máximo & Variância & $\begin{array}{c}\text { Des vio } \\
\text { padrão }\end{array}$ & $\begin{array}{c}\text { Coef. de } \\
\text { variação, \% }\end{array}$ \\
\hline Açúcar redutor total, g/100 mL & 7 & 10,48 & 11,51 & 12,94 & 0,93 & 0,96 & 8,37 \\
Açúcar redutor solúvel, g/100 mL & 7 & 5,67 & 7,78 & 10,48 & 3,00 & 1,73 & 22,27 \\
Frutose, g/100 mL & 7 & 3,90 & 6,12 & 8,96 & 2,90 & 1,70 & 27,85 \\
Glucose, g/100 mL & 7 & 1,40 & 1,66 & 2,06 & 0,05 & 0,22 & 13,28 \\
Sacarose,g/100 mL & 7 & 0,00 & 3,73 & 6,20 & 4,89 & 2,21 & 59,30 \\
Frutose/glucose* & 7 & 2,21 & 3,74 & 5,86 & 1,27 & 1,13 & 30,17 \\
Acidez, g/100 mL & 7 & 0,20 & 0,30 & 0,38 & 0,00 & 0,06 & 18,72 \\
Acidez/ART* & 7 & 31,54 & 40,61 & 57,96 & 88,62 & 9,41 & 23,18 \\
Cor & 7 & 0,22 & 0,35 & 0,52 & 0,01 & 0,10 & 28,66 \\
Fenóis totais, mg/L & 7 & 192,66 & 356,31 & 623,76 & 23321,01 & 152,71 & 42,86 \\
Nitrogênio, mg/L & 7 & 87,62 & 130,68 & 173,94 & 929,15 & 30,48 & 23,33 \\
\hline
\end{tabular}

Nota: * adimensional 
A acidez titulável total apresenta-se como um atributo de grande expressão na discriminação de cultivares de maçã, sendo importante na valoração de sucos; aliás, somente se encontram no mercado industrial de sucos concentrados de alta acidez (DOWNING, 1989). Os resultados das análises evidenciaram um valor médio de $0,30 \pm 0,06 \mathrm{~g} / 100$ $\mathrm{mL}$, com uma variação da ordem de $18,72 \%$. O valor médio encontrado encontra-se abaixo de $0,45 \mathrm{~g} / 100$ $\mathrm{mL}$ preconizado na literatura como limite entre as maçãs doces e as ácidas (LEA, 1995). Assim, de uma forma generalizada, pode-se considerar que o conjunto de genótipos deste estudo se caracteriza como doce.

Para o atributo cor, houve um pequeno desvio padrão de 0,10 , sendo que o valor médio foi de 0,35 , com um coeficiente de variação de $28,66 \%$. O conjunto analisado apresentou um teor médio de compostos fenólicos de $356,31 \pm 152,71 \mathrm{mg} / \mathrm{L}$, havendo um coeficiente de variação superior a $40 \%$, ou seja, há bastante heterogeneidade com relação a este parâmetro.

O valor médio para o nitrogênio foi de 130,68 \pm $30,48 \mathrm{mg} / \mathrm{L}$, com um coeficiente de variação de
$23,33 \%$, que qualifica como um mosto sem carência nesse elemento para um processo fermentativo.

As maçãs comerciais podem ser classificadas de acordo com os valores de açúcares, de acidez total e de compostos fenólicos, como preconizado na literatura (SMOCK; NEUBERT, 1950; BEECH; CARR, 1977; DRILLEAU, 1991; LEA, 1995). Em se considerando os teores de açúcares totais superiores a 11,5 g/100 mL, as maçãs são consideradas como frutas interessantes para o processamento da sidra. A relação ART/acidez total é considerada como um indicador tanto para uso industrial como para consumo in natura. Esta relação tem o valor 20 como delimitante para as duas opções. Desta forma, as cultivares com valores abaixo de 20 apresentam interesse industrial e acima deste valor, as frutas são adequadas ao consumo in natura . Na Figura 1 , pode ser visto que apenas a média do RSK ficou abaixo do valor 20. É importante salientar que todas as cultivares e seleções objeto deste estudo apresentaram valores de relação ART/Acidez acima deste valor e; isto indica que estes genótipos apresentaram-se adequados para o consumo in natura, numa comparação com o valor de RSK apresentado.

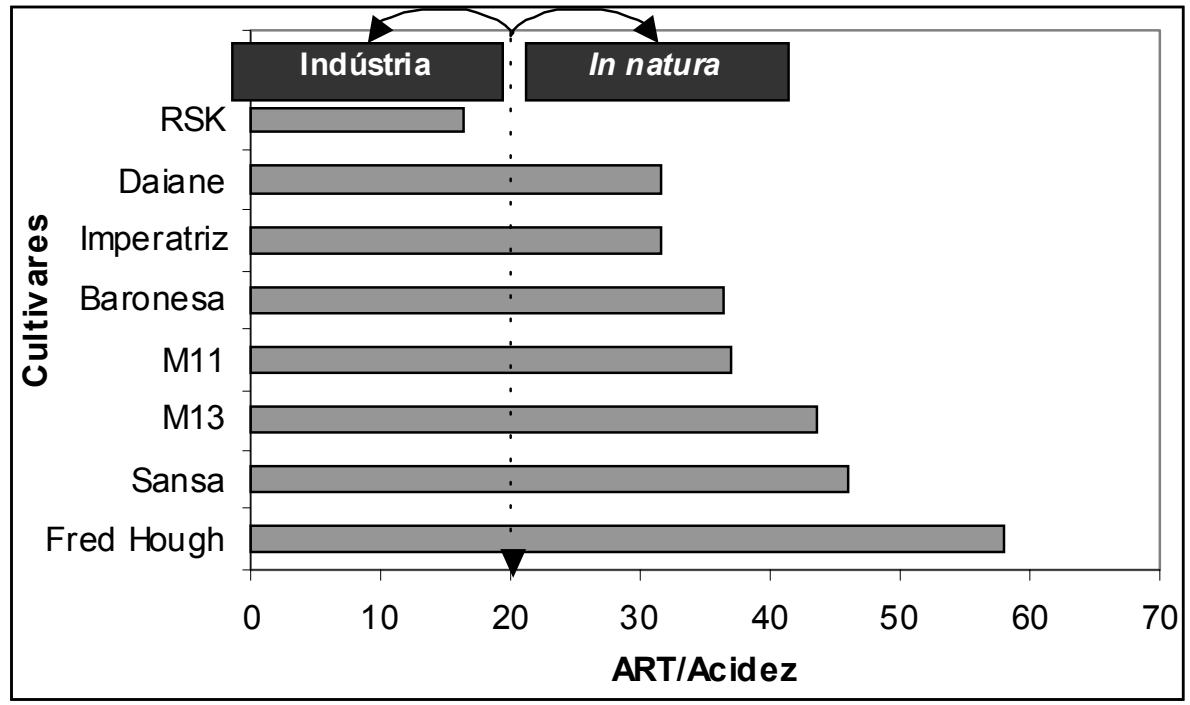

Figura 1. Classificação de quatro novas cultivares e duas seleções de macieira desenvolvidas no Sul do Brasil pela Epagri em termos de teores de açúcares totais e ácidos em relação à cultivar japonesa Sansa. 
Segundo a proposta de Blends (apud PROULX; NICHOLS (1980), [1] maçãs doces com baixo teor de acidez podem ser utilizadas para obtenção de um suco básico; [2] as com elevado teor de ácidos, para a sidra, sendo que as de aroma pronunciado podem melhorar o 'flavor' e o 'bouquet' do produto; [3] as frutas com elevado teor de fenóis podem melhorar o 'corpo' e o 'flavor' dos produtos, na proporção de $10 \%$ para mostos ácidos e $20 \%$ para mostos com baixa acidez.

Outra forma de classificação é através dos limites de 200 ppm para os compostos fenólicos e $0,45 \mathrm{~g} /$
$100 \mathrm{~mL}$ para a acidez. Desta forma, as cultivares podem ser discriminadas em doces-amargas; doces; amargas-ácidas e ácidas (LEA, 1995; DRILLEAU, 1991). A Figura 2 mostra esta classificação, indicando que as cultivares Sansa, Imperatriz, Daiane, Baronesa e a seleção M-11/00 se encontram no primeiro quadrante, correspondendo às doce-amargas, enquanto que cv. Fred Hough situou-se na linha delimitante entre as doce-amargas e as doces; já a M-13/00 enquadrouse no terceiro grupo, significando tratar-se de um genótipo com características mais doces.

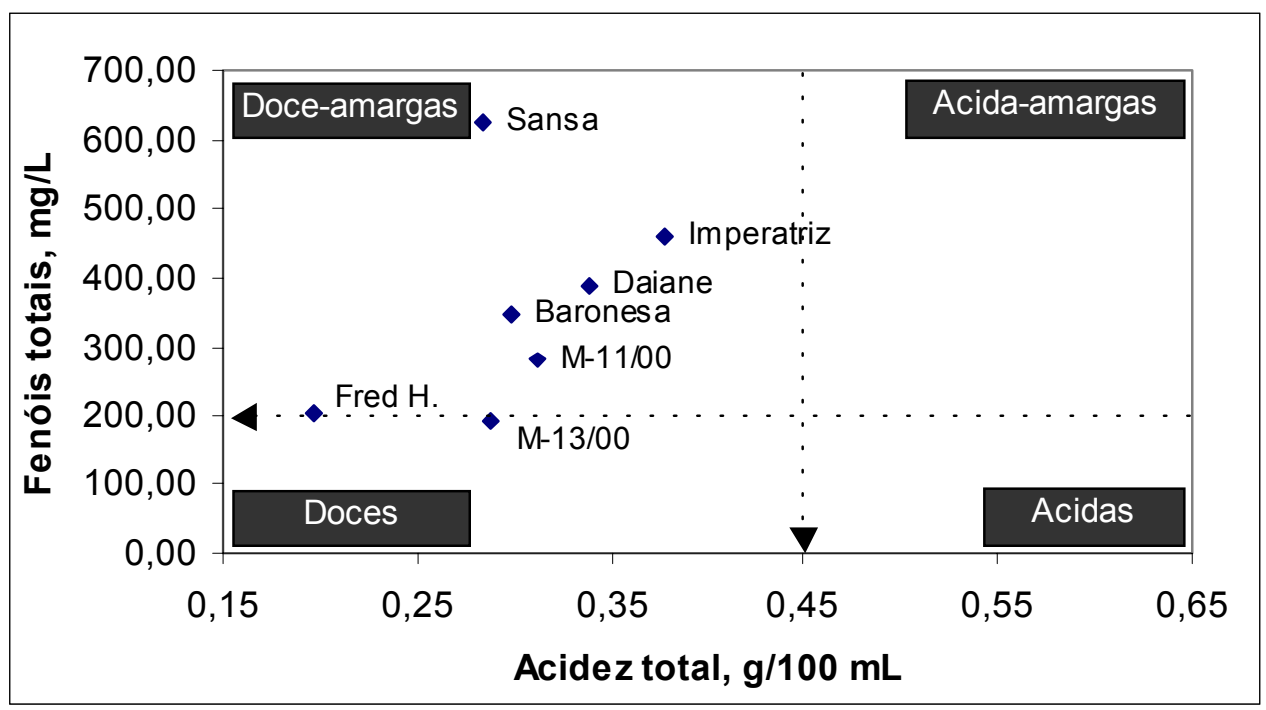

Figura 2. Enquadramento de quatro novas cultivares e duas seleções de macieira desenvolvidas no Sul do Brasil pela Epagri em termos de sabor comparativamente à cv. Sansa.

\section{Conclusão}

Os valores médios das cultivares apresentaram diferenças significativas em todas as análises realizadas, salvo os teores de ácidos, cujos valores não se diferenciaram ao nível de 5\%. As cultivares apresentaram baixos teores de ácidos, sendo caracterizadas como doces e doce-amargas, atributos interessantes para o comércio de frutas in natura. A cv. Sansa apresentou destaque no teor de açúcares e fenóis totais, podendo ser utilizada em 'blends' e cortes no processamento de sucos e sidras. Estas frutas apresentam características para o comércio visando o uso in natura e trabalhos devem ser realizados para sua valorização como matéria-prima industrial.

\section{Agradecimentos}

Os autores são gratos à EPAGRI pelo envio das amostras, à NOVOZIMES do Brasil pela doação das enzimas, e aos órgãos de fomento à pesquisa CNPq e CAPES pelo apoio recebido para o desenvolvimento dos projetos que deram origem a este trabalho, feito sob a égide da UEPG por integrantes do Grupo de Trabalho sobre Maçã www.uepg.br/gtm. 


\section{Referências}

AGÊNCIA NACIONAL DE VIGILÂNCIA SANITÁRIA. Lei $n .^{\circ}$ 14.242, de 29 de julho de 2002, DOE de 05.08.2002. Portaria $n^{\circ}$ 29/28/SVS/MS, do dia 13/01/ 1998. Disponível em: <http://www4.anvisa.gov.br/base/ visadoc/LEI/LEI\%5B2705-1-0\%5D.HTM > . Acesso em: 16 fev. 2005.

ASSOCIAÇÃO BRASILEIRA DE PRODUTORES DE MAÇÃ. Dados estatísticos sobre a cultura da macieira. Disponível em: $<$ http://www.abpm.org.br $>$.Acesso em: 2 fev. 2005.

ASSOCIATION OF OFFICIAL ANALYTICAL CHEMISTS. Official Method of Analysis. Washington, 1965.

BARON, A.; BOHUON, G.; DRILLEAU, J. F. Remarques sur l'indice formol des concentrés de jus de pomme. Annales des Falsifications et de l'Expertise Chimique, Paris, v.70, n.749, p.19-26, 1977.

BEECH, F. W.; CARR, J. G.; PERRY, C. In: ROSE, H. A (Ed.). Economic microbiology. London: Academic Press, 1977. Cap.139-313.

CAMILO, A. P.; DENARDI, F. Cultivares: descrição e comportamento no sul do Brasil. In: A cultura da macieira. Florianópolis: EPAGRI., 2002.

CZELUSNIAK, C.; OLIVEIRA, M. C. S.; NOGUEIRA, A.; SILVA, N.C.; WOSIACKI, G. Qualidade de maçãs comerciais produzidas no Brasil - Aspectos físicosquímicos. Brazilian Journal of Food Technology, Campinas, v.6, p.25-31, 2003.

DEL CAMPO, G.; SANTOS, J. I.; BERREGI, I.; MUNDUATE, A. Differentiation of Basque cider apples juices from different cultivars by means of chemometric techniques. Food Control, Guildford, v.16, n.6, p.551-557, 2004.

DENARDI, F.; CAMILO, A. P. Daiane: nova cultivar de macieira para colheita em março. Revista Agropecuária Catarinense, Florianópolis, v.11, n.3, p.6-8, 1998.

Epagri 404 - Imperatriz: nova cultivar de macieira para dupla finalidade - produtora e polinizadora. Revista Brasileira de Fruticultura, Cruz Das Almas (BA), v.22, n.1, p.40-43, 2000.

. Epagri 406: Baronesa: nova cultivar de macieira de maturação tardia para o sul do Brasil. Revista Brasileira de Fruticultura, Cruz Das Almas (BA), v.19, n.2, p.185189, 1997a.

. Nova cultivar de macieira com imunidade à sarna. Revista Brasileira de Fruticultura., Cruz Das Almas (BA), v.16, n.1.p.1-6, 1994.
. Novas cultivares de macieira: proposta de nova composição de pomares com polinizadoras/produtoras. Revista Agropecuária catarinense, Florianópolis, v.10, n.2, p.25-30, jun. 1997b.

DOWNING, D. L. Apple cider. In: DOWNING, D. L. Processed apple products. New York: Van Nostrand Reinhold, 1989. p.169-187.

DRILLEAU, J. F. Produits cidricoles. quelques mots sur les composés phénoliques (tanins). Technologie, Paris, v.23, p.21-22, 1991.

DUTCOSKI, S. D. Analise sensorial de alimentos. Curitiba: Univ. Champagnat, 1996.

EMPRESA DE PESQUISA AGROPECUÁRIA E EXTENSÃO RURAL DE SANTA CATARINA. A cultura da macieira. Florianópolis: GMC/Epagri, 2002.

FEATHE, W. RSK- Wert, Die Gesamtdarstellung: Verband Derdeutscherfruchtsaft Industrie e Bonn. Verlag Flüssiges: Obst $\mathrm{GmbH}, 1987$

IAL. Métodos físicos e químicos para análise de alimentos. In: _ Normas Analíticas. 3.ed. São Paulo: Instituto Adolfo Lutz, 1976.

KENNEDY, M.; LIST, D.; LU, Y.; FOO, L. Y.; NEWMAN, R. J.; SIMS, I. M.; BAIN, P. J. S.; HAMILTON, B.; FENTON, G. Apple pomace and products derived from apple pomace: uses, composition and analysis. In: LINSKENS, G.; JACKSON, J. F. Modern methods of plant analyses: analysis of plant waste materials. Berlin, Heidelberg: Springer Verlag, 1999. v.20, p.5-119,

LEA, A. G. H. Cidermaking. In: LEA, A. G. H.; PIGGOTT, J. R. Fermented beverage production. Glasgow: Blackie and Sons, 1995. p.66-96.

NAMIUCHI, N. N.; WOSIACKI, G.; ROCHA, M. A. Avaliação dos sucos de cinco variedades de maçãs nacionais: características sensoriais dos sucos das variedades Fuji, Gala, Golden Delicious, Granny Smith e Starkrimson. Revista Cientifica e Cultural da Universidade Federal de Mato Grosso do Sul, Campo Grande,v.7, n.1, p.15-22, jan. 1992.

NOGUEIRA, A.; OLIVEIRA,R.G.; DENARDI,F.; WOSIACKI,G. Características físico-químicas de 103 cultivares de macieira analisadas nas safras de 1984 a 2004. In: CONGRESSO BRASILEIRO DE CIÊNCIA E TECNOLOGIA DE ALIMENTOS, 19., 2004, Recife. Anais... Recife: SBCTA, setembro 2004.

NOGUEIRA, A.; WOSIACKI, G. S. In: VENTURINIFILHO, W.G. Tecnologia de bebidas: matéria-prima, processamento, BPF/APPCC, legislação, mercado. Botucatu: Blücher, 2005. p.383-422 
PROULX, A.; NICHOLS, L. Sweet and hard cider. Missouri: Garden Way Publishing, 1980.

SINGLETON, V. L.; ORTHOFER, R.; LAMUELARAVENTOS, R. M. Analysis of total phenols and other oxidation substrates and antioxidants by means of Folin-Ciocalteu reagent. Methods in Enzymology, New York, v.299, p.178-184, 1999.

SMOCK, R. M.; NEUBERT, A. M. Apples and apples products. New York: Interscience Publishers, 1950.

TANNER, H.; BRUNNER, H. R. Getränke Anlytikuntersuchungsmethode für dia Labor- und Betriebspraxis. Wädesnwill: Verlag Helles, 1985.
WOSIACKI, G.; NAMIUCHI, N. N.; CERIBELI, M. I. P. F.; SATAQUE, E. Y.; SICHIERI, V. L. F. S.; OLIVEIRA, T. C. R. M.; OLIVEIRA CESAR, E. Estabilidade do suco clarificado de maçã. Arquivos de Biologia e Tecnologia, Curitiba, v.32, n.4, p.775-786, 1989.

WOSIACKI, G.; NOGUEIRA, A.; SILVA, N. C. C.; DENARDI, F.; CAMILO, A. P. Apple varieties growing in subtropical areas - the situation in Santa Catarina-Brazil. Fruit Processing, Schönborn: v.12, n.1, p.19-28, 2002.

WOSIACKI, G.; NOGUEIRA, A.; Suco de maçã. In: VENTURINI FILHO, W.G. Tecnologia de bebidas: matéria-prima, processamento, BPF/APPCC, legislação, mercado. Botucatu: Blücher, 2005. p.255-292. 